\title{
The Role of Biobanks in Oncology Research - A Portuguese Perspective
}

\author{
Mónica Gomes $s^{1,2,3, \#}$, Ana Luísa Teixeira ${ }^{1,2,3, \#}$, Ana Coelho ${ }^{1,2,3,4}$, Rui Medeiros ${ }^{1,3,6}$ and \\ António Araújo ${ }^{2,5, *}$
}

\author{
${ }^{1}$ Molecular Oncology and Viral Pathology GRP - Cl, Portuguese Institute of Oncology, Porto, Portugal \\ ${ }^{2}$ Unit of Oncobiology Research- Unit for Multidisciplinar Research In Biomedicine, Abel Salazar Institute for \\ the Biomedical Sciences, University of Porto, Porto, Portugal (UNIO-UMIB-ICBAS) \\ ${ }^{3}$ LPCC Research Department-Portuguese League Against Cancer (NRNorte) Porto, Portugal \\ ${ }^{4}$ Faculty of Medicine of University of Porto, Portugal \\ ${ }^{5}$ Medical Oncology Service of Centro Hospitalar do Porto, Porto \\ ${ }^{6}$ Faculty of Health Sciences of Fernando Pessoa University, Porto, Portugal
}

\begin{abstract}
Approximately, 14.1 million new cancer cases and 8.2 million deaths occurred in 2012 worldwide. Cancer is a group of diseases characterized by uncontrolled growth, spread of abnormal cells and is a leading cause of death in both more and less economically developed countries. The growth in our knowledge of cancer biology has led to remarkable progress in cancer prevention, early detection and treatment. Recent years have seen a remarkable progress in the basic, translational and clinical research in cancers. During carcinogenesis several genetic and epigenetic alterations occur and a molecular approach based on the detection of these molecular alterations can permit the definition of molecular signatures specific for different tumors, allowing the definition of molecular biomarkers. These markers can be found in a variety of fluids, tissues and cell lines, and can be used for screening the general population, for differential diagnosis in symptomatic patients and for clinical staging of cancer. Furthermore, the biomarkers can be used to evaluate response to treatment, to assess disease recurrence through monitoring and as prognostic indicators of disease progression. The advances in the molecular knowledge and technology from research is based on the study of different types of biological samples, being necessary the collection, processing, preservation, stored and maintenance of these samples required an ordered and structured plan with the consequent creation of a biobank. The promotion and operation of biobanks provide to scientists the opportunity to access to quality samples and associated data, representing an essential milestone in shortening the time between research and clinical application, improving the effectiveness of research.
\end{abstract}

Keywords: Biobank, cancer research, cancer biomarkers.

\section{INTRODUCTION}

Cancer is a group of diseases (more than 200) characterized by uncontrolled growth, spread of abnormal cells and is a leading cause of death in both more and less economically developed countries. The occurrence of cancer is increasing because of the growth and aging of the population, as well as an increasing prevalence of established risk factors such as smoking, overweight, physical inactivity, and changing reproductive patterns associated with urbanization and economic development. About 14.1 million new cancer cases and 8.2 million deaths occurred in 2012 worldwide [1]. Over the years, the burden has shifted to less developed countries, which currently account for about $57 \%$ of cases and $65 \%$ of

${ }^{*}$ Address correspondence to this author at the Unit of Oncobiology ResearchUnit for Multidisciplinar Research In Biomedicine, Abel Salazar Institute for the Biomedical Sciences, University of Porto, Porto, Portugal;

Tel: +351222077500; Fax: +351222086085;

E-mail: antonio.araujo@chporto.min-saude.pt

"Contributed equally to this review. cancer deaths worldwide [1]. Lung cancer is the leading cause of cancer death among males in the world, and has surpassed breast cancer as the leading cause of cancer death among females in more developed countries [1].

The growth in our knowledge of cancer biology has led to remarkable progress in cancer prevention, early detection and treatment. Recent years have seen a remarkable progress in the basic, translational and clinical research in cancers. Over the last decades, the Oncology field was a target of significant advances in the knowledge of the biology of carcinogenesis processes. Several discoveries were made and the identification of key genes and molecules in the regulation of cancer growth, differentiation, apoptosis inhibition, invasion and metastasis development has dramatically changed the view of the oncology [2]. Molecular mechanism of cancer involve a several number of genetic alterations, however only a limited part of them are found in all tumor types, which could 
be key alterations for oncogenesis and tumor progression [3].

The oncology community has seen a paradigm shift from the histology to the molecular diagnosis and treatment of cancer thanks to identification of genetic markers, which are more reproducible and less subject to intrinsic and extrinsic stimuli [4]. The personalized medicine (as a form of medicine that uses information about a person's genes, proteins and environment to prevent diagnose and treat disease), has changed the oncology field, in consequence of being based on understating molecular carcinogenesis, pharmacogenomics and individual genetic differences that determined the response to anti-neoplastic agents. Nowadays, the use of molecular biomarkers in oncology, that provide molecular information regarding the tumor biology, are useful tools for cancer signatures, to personalized treatment and can be divided to: diagnostic, prognostic, treatment and prevention subgroups [4].

\section{MOLECULAR BIOMARKERS}

Biomarkers can be defined as biological molecules found in blood, other body fluids or tissue that is a sign of a normal or abnormal process, or of a condition or a disease [5]. These biomarkers can typically differentiate an affected patient from a person without the disease. This differentiation could be based in several types of alterations, including germline or somatic mutations, transcriptional changes and posttranslation modification. Thus, a large number of biomarkers could include proteins, nucleic acids and antibodies. Moreover, a biomarker can also be a collection of alterations such as gene expression, proteomic, and metabolomics signatures and being detected in circulation (whole blood, serum or plasma), excretions or secretion (stool, urine, sputum or nipple discharge) and could be easily assessed noninvasively [5]. By definition, an ideal cancer biomarker is characterized by the specificity for a given type of tumor with an appropriated level of sensibility, and the concentration of the biomarker should indicate the stage of the disease and the response to treatment used [3].

Recently, numerous studies have demonstrated that several molecular markers can assist to predict prognosis in cancer. During carcinogenesis several genetic and epigenetic alterations occur and a molecular approach based on the detection of these molecular alterations can permit the definition of molecular signatures specific for different tumors, allowing the definition of molecular biomarkers [6].

Biomarkers can be used for patient's assessments in multiple clinical settings in the continuum of the disease, including risk, screening, diagnostic, determining prognosis and prediction of clinical outcomes and treatment response.

Biomarkers can be used to found an individual risk for cancer development. One example is the germline mutation in BRAC1 gene, which increases the risk of breast and/or ovarian cancer development. Women with a strong family history of ovarian and breast cancer can undergo genetic testing to determine if she is a carrier of these mutations [5]. In positive cases, women can opt for a more intensive screening, chemoprevention with tamoxifen or prophylactic bilateral mastectomy and/or bilateral salpingooophorectomy in order to decrease the risk of develop these cancers $[5,7]$.

Another approach, is use biomarkers to screen otherwise healthy individuals for diseases, a common but controversial biomarker for screening is prostate specific antigen (PSA). The use of this biomarker was approved in 1986 by Food and Drug Administration (FDA) and is responsible for an increase in the diagnosis of prostate cancer (PC), but also led to a PC overdiagnosis and overtreatment [8].

Information provided by diagnostic biomarkers makes possible quicker and differential diagnosis. If in a patient was found a suspicious nodule in breast on mammography, histological evaluation of the biopsy specimen can determine whether the tissue is cancer, infection, inflammation or another benign process. If cancer, the tissue will be submitted to evaluation with specific immunohistochemical markers for the identification of the origin of the tumor $[5,9]$.

After patients being diagnosed with cancer, biomarkers could have a prognostic role (likelihood of disease recurrence independent of treatment) and/or a predictive role (help determining a more accurate treatment choice).

Classically, the clinicopathological characteristics of tumor have been used for determination of prognosis. Nowadays, newer technologies and approaches are being used to assess prognosis for individual tumors, 
changing the clinical practice. In breast cancer there are a number of gene expression profiles that have been developed and that can be used to estimate prognosis for an individual patients based on the assessment of the tumor. More recently, in metastatic breast cancer the use of circulating tumor cells (CTCs) show to be prognostic for overall survival $[5,9]$. On the other hand, the predictive biomarkers provide information on the probability of obtaining a response to treatment and support the process of therapeutic decision. It has been proved that somatic mutation in exons 19 or 21 of EGFR gene are related to tumor sensitivity to TK is therapies in lung cancer $[4,6]$. In colorectal cancer, mutation of the gene RAS is a predictive biomarker, because RAS somatic mutations are associated with poor response to target therapy using antibodies anti-EGFR [5].

Thereby, biomarkers can be found in a variety of fluids, tissues and cell lines, and can be used for screening the general population, for differential diagnosis in symptomatic patients and for clinical staging of cancer. Furthermore, the biomarkers can be used to evaluate response to treatment, to assess disease recurrence through monitoring and as prognostic indicators of disease progression [9]. However, only a few markers are used in routine practice and are limited to cancer type and clinical setting [5].

The definition of new potential biomarkers can be identified through different approaches. The traditional view identifies candidate biomarkers based on the biology of the tumor and microenvironment, or the metabolism of the pharmaceutical drugs. In the new Era of molecular biology, the biomarker identification use techniques such as high-throughput sequencing, gene expression arrays and mass spectrocospy will permit to quickly identify individual or groups of biomarkers. However, for the evaluation in the daily clinical practice of a patient and for the study of new potential biomarkers, is imperative the use of biological individual samples, which can be security persevered with a quality guaranteed $[5,9]$. Thus, is imperative the construction of biobanks for the present and for the future research development in this field.

\section{EVOLUTION OF THE PATIENT'S TREATMENT BASED ON MOLECULAR SIGNATURES}

During the 1970s approximately 1 of 2 people diagnosed with cancer survived at least 5 years, nowadays more than 2 of 3 survive. Until the late 1990s nearly all drugs used in cancer treatment (with the exception of hormone treatment) worked by killing cells that were in the process of replicating their DNA and dividing to form new cells. These anti-neoplastic drugs also killed normal cells but had a greater effect in cancer cells because their turnovers were greater. During 1980s scientists observed that several growth factors and other molecules responsible for recognizing and responding to growth factors are actually products of oncogenes. Among the earliest targeted therapies that block signals are trastuzumab, gefitinib, imatinib and cetuximab [7].

Personalized therapy based on patient's individual biologic and molecular profiles is a promising approach to optimize efficacy with the available agents. The target therapy use agents that affect a known aberrant signal pathway or molecular target in cancer cell or tumor microenvironment [10]. The initial studies of target therapies included the use of trastuzumab to treat HER2-amplified breast cancer and imatinib for BCR-ABL translocated chronic myelogenous leukemia. In the last decade, it was observed an increase in clinical trials of drugs targeting oncoproteins and cancer pathways in several solid tumors $[3,7,10]$.

A clear example of this field includes agents targeting receptors tyrosine kinase, such as the EGFR tyrosine kinase inhibitors (gefitinib, erlotinib or afatinib) for lung cancer treatment. Lung cancer is also a paradigmatic example of advances in patient's treatment - in the past, treatment plans have been prescribed to patients based on clinicopathological criteria [11], but in nowadays, new treatments take into account the histology, genetic and molecular characteristics of the tumor as well as patient's characteristics [12]. Thus, molecular subtyping of NSCLC has led to approval of and use of targeted therapies in the front-line setting (gefitinib, erlotinib, and afatinib for EGFR; crizotinib for ALK and ROS1) [13].

In 2004, EGFR mutations were detected in patients with lung adenocarcinoma and these patients responded better to tyrosine kinase inhibitor (TKI) treatment - a new era of targeted therapy in NSCLC has begun [2]. Mutation of EGFR has been detected in a small subset of NSCLC patients, more frequent in the Asian than in the Caucasian population, more frequent in non-smokers than in smokers and more frequent in adenocarcinoma histology. These are activating 
mutations found in exons $18,19,20,21$ of the EGFR gene. Four randomized phase III trials with gefitinib, two randomized phase III trials with erlotinib, and one randomized phase III trial with afatinib prospectively compared the efficacy of EGFR TKis against standard platinum-based chemotherapy in patients with EGFR mutations positive NSCLC. In all trials, it was observed that EGFR-mutated NSCLC patients treated with TKis had significantly better progression-free survival (PFS) and quality of life (QOL) when compared with patients treated with platinum-based chemotherapy [13].

In 2007, Soda and co-workers, identified a small inversion in the $p$ region of chromosome 2 in NSCLC patients, whose consequence was the formation of the fusion gene EML4-ALK. This translocation occurs in different histological types of NSCLC, in non-smoking patients and most often does not occur together with the mutations in the EGFR and KRAS genes [3, 14]. The phase III randomized trials with crizotinib compared to standard chemotherapy in patients with this gene alteration showed a statistically improved PFS with the use of target therapy $[15,16]$.

These evidences support the assessment of mutations in the EGFR gene and the EML4-ALK fusion gene that may be used as a predictive biomarker in NSCLC.

\section{BIOBANKS: OVERVIEW}

Over the years, the biomedical research improves the population health care. It was observed a significant increase of the studies that combined basic science and the clinic, opening a new field - the translational research. This research will be critical to improve the prevention, detection, diagnosis, intervention and treatment, including the development of new molecular tests [6, 9].

The translational research combine the molecular knowledge of basic science with clinical information, improving efficient patient's health care [17]. However, to get this aim it is necessary advances in the molecular knowledge and technology from research based on the study of different types of biological samples. The collection, processing, preservation, stored and maintenance of these samples required an ordered and structured plan with the consequent creation of a biobank.

Biobanks could be defined as storage banks for human origin samples, usually associated with personal information, which together allow the national and international biomedical research [17, 18]. For more than 80 years tissue has been derived from human bodies, stored and used for therapeutic, educational, forensic and research purposes in most western countries [19]. Nowadays, we observed a rapid proliferation and an increase in complexity of biobanks, in consequence of diverse research purposes and types and sources of samples. Thus, it is important that biobanks bear a large number of representative samples of biological tissues, tumor cells, proteins, DNA, RNA, miRNAs and other vital fluids such as blood, serum, urine, pleural or ascitic fluid. Regarding the nucleic acids and proteins is advice that in collection and isolation be used the same tissue specimen, because tumor heterogeneity is one of the major sources of variation in biological data [20]. Taken together, the recent advances in genomic and proteomics (and other "omics") allow a better understands of the molecular characteristics of these samples. This new Era enabled a large amount of information data, ensuring the quality and the rapid availability.

According to the guidelines on Human Biobanks and Genetic Research Databases (HBGRD) established on October 2009, from Organization for Economic Co-Operation and Development (OECD) who helps Governments response to new development and concerns, the biobanks must be focus on the management, under safety, quality and efficiency criteria of samples.

According the principles defined by OECD, the HBGRD objective should be to foster research, as well as should be established governed, managed, operated, accessed, used and discontinued in accordance with applicable legal frameworks and ethical principles. Moreover, the HBGRD operators should strive to make data and material rapidly and widely available to researchers to advance knowledge and understanding, however, these operators and users should respect human rights, freedoms and secure of participants' privacy and the confidentiality of data and information. The operators should also considered and minimize risks to participants, their families and potentially identifiable populations or groups whose specimens and data are included in HBGRD. Regarding the collection, labeling, registration, processing, storage, tracking, retrieval, transfer, use and destruction of human biological 
materials, data and/or information, the operators should documented all operating procedures and policies. Moreover, the HBGRD operators should be transparent and clear regarding the nature and source of funding. As final work, the use of samples from biobanks should result in scientific publications and/or the final results must be available for all scientific community.

The biobank creation should be based on rules clearly formulated and communicated, should have professional and qualified staff for all operations, that should be efficiently performed, and should have defined a long term sustainability strategy. These guidelines provide information regarding the establishment; governance; management; oversight; terms of participation; type of biological sample; protection of human biological materials and data; access; qualifications, education and training of operators; custodianship, benefits, sharing and intellectual property; discontinuation and disposal material and data [21].

However, the creation of biobanks rises up several ethical issues [18]. The use of human biological samples can be considered as an invasion of the individual autonomy, with no self-control of the process. According to Budimir and co-workers, several questions can be made, such as - When individuals donate part of their body to a biobank how is that human sample processed? Who is the owner of the sample? Who should decide how it should be used? Who has the right to know individual results of research?

Regarding biobanks, ethical framework has been the most controversial issue. For example, the type of the study should be considered, because the epidemiological studies results will not induce the same levels of relevance as the results of genetic information. Similarly, the collection of cancer samples is not associated with the same levels of concern as for example collecting of the samples to be used in the creation of stem cells that will be transplanted into humans. Another example regarding the relevance of ethical issue is the increase of published articles related to ethics in biobanks in the last 12 years [18].

Particularly in Portugal, the main problem associated with the ethical issue of biobanks is the obtainment of informed consent. Most biological specimens stored in tumor biobanks were only collected for histopathological cancer diagnosis, however in recent years the investigators realized that the additional molecular analysis of these samples and their correlation with the obtained clinical results could be useful for translational researches. Therefore is crucial to find a good strategy for obtaining informed consent to use specimens already collected for new translational studies.

Other important issues for biobanks creators are the accuracy of clinical information and outcome of the cancer patients treated, and the quality of tumor samples. Thus, a biobank with an effective and reliable protocol allow researchers to stores and access tumor tissue samples under ideal conditions for their researches and recovery of patient's data [22].

The ethical problems, involving all aspects discussed above, will never have an easy answer and are constantly evolving, in consequence of the huge advances in genetic research together with the development of science and technology. In the future, the biobanks need to protect patients sensitive information, levels of accessibility, the need to prevent data misuse and the possibility to predict individual health-related outcomes based on the genetic/genomic information [18].

\section{THE ROLE OF BIOBANKS IN CANCER RESEARCH}

The Oncology is a wide field, with etiologic and pathophysiologic mechanisms mostly unknown or not completely understood. It is necessary to develop new therapies using genetic studies that could better characterize the patient's tumors and their carcinogenesis evolution, as well as individual's genetics characteristics.

The biobanks should offer samples from various sources of each patient (tissue or fluid) and at different times of disease progression (diagnosis, progression, pre or pos-treatment) to may help to clarify the mechanisms involved in cancer development. The researchers can take advantage of the existence of a biobanks to propose the identification of new biomarkers helpful in the identification of new target molecules for the development of drugs and new therapies. Biobanks can thus have an important role on biomedicine research and decisively contribute to the development of the personalized medicine [17].

The promotion and operation of biobanks provide to scientists the opportunity to access to quality samples 
and associated data, representing an essential milestone in shortening the time between research and clinical application, improving the effectiveness of research.

Nevertheless, it is important to have constant coordination and collaboration between biobanks with different professional involved in the processing of samples and the information associated with them: identification and phenotyping of donors, acquisition, processing, storage, distribution and use of samples and associated information [17]. To promote the development of effective cancer treatments, scientists increasingly rely on human tissue specimens for preclinical drug discovery. The existence of a biobank well-structured and with quality organic samples is an essential part of the complex, lengthy and expensive process for cancer drug development.

The use biobanks in cancer research is crucial to: 1) evaluate patients responses and their correlation with the clinic information (most studies using animal models for drug analysis are inefficient); 2) molecular analysis, including comparative genomic and quantified gene expression; 3) a higher number of specimens (high representatively of the disease); 4) prospective sample collection useful for drug discovery efforts; 5) high quality procedures for sample collection, storage and analysis; 6) maintained the confidential of the samples and the patients information. These procedures are basal for an ideal translational research, allowing an improvement of patient's quality of life and increasing overall survival.

In conclusion, only research biobanks provides the expertise, infrastructure and resources to provide the research community with appropriate collection of biological samples. Only biobanks provide the opportunity to biomarkers identification based on rigorous scientific studies and tested in focused welldesigned clinical trials, allowing a more efficient clinical development. For patients the use of these data represents enormous benefits in consequence of the development of more efficacious and less toxic therapies, improving the overall survival [7].

\section{REFERENCES}

[1] Torre LA, Bray F, Siegel RL, Ferlay J, Lortet-Tieulent J, Jemal A. Global cancer statistics, 2012. CA: a cancer journal for clinicians 2015 ; 65(2): 87-108.

http://dx.doi.org/10.3322/caac.21262
Popper HH, Ryska A, Timar J, Olszewski W. Molecular testing in lung cancer in the era of precision medicine. Translational lung cancer research 2014; 3(5): 291-300.

[3] Nalejska E, Maczynska E, Lewandowska MA. Prognostic and predictive biomarkers: tools in personalized oncology. Molecular diagnosis \& therapy 2014; 18(3): 273-84. http://dx.doi.org/10.1007/s40291-013-0077-9

[4] Kalia M. Biomarkers for personalized oncology: recent advances and future challenges. Metabolism 2015; 64(3 Suppl 1): S16-21. http://dx.doi.org/10.1016/j.metabol.2014.10.027

[5] Henry NL, Hayes DF. Cancer biomarkers. Molecular oncology 2012; 6(2): 140-6. http://dx.doi.org/10.1016/j.molonc.2012.01.010

[6] Sethi S, Ali S, Philip PA, Sarkar FH. Clinical advances in molecular biomarkers for cancer diagnosis and therapy. International journal of molecular sciences 2013; 14(7): 14771-84.

http://dx.doi.org/10.3390/ijms 140714771

[7] La Thangue NB, Kerr DJ. Predictive biomarkers: a paradigm shift towards personalized cancer medicine. Nature reviews Clinical oncology 2011; 8(10): 587-96. http://dx.doi.org/10.1038/nrclinonc.2011.121

[8] Shariat SF, Scherr DS, Gupta A, Bianco FJ, Jr., Karakiewicz $\mathrm{PI}$, Zeltser IS, et al. Emerging biomarkers for prostate cancer diagnosis, staging, and prognosis. Arch Esp Urol 2011; 64(8): 681-94

[9] Kulasingam V, Diamandis EP. Strategies for discovering novel cancer biomarkers through utilization of emerging technologies. Nature clinical practice Oncology 2008; 5(10): 588-99.

http://dx.doi.org/10.1038/ncponc1187

[10] Aggarwal C. Targeted therapy for lung cancer: present and future. Annals of palliative medicine 2014; 3(3): 229-35.

[11] Song G, Liu Y, Wang Y, Ren G, Guo S, Ren J, et al Personalized biomarkers to monitor disease progression in advanced non-small-cell lung cancer patients treated with icotinib. Clin Chim Acta 2015; 440: 44-8. http://dx.doi.org/10.1016/j.cca.2014.11.010

[12] Vadakara J, Borghaei H. Personalized medicine and treatment approaches in non-small-cell lung carcinoma. Pharmacogenomics and personalized medicine 2012; 5 : 113-23.

[13] Korpanty GJ, Graham DM, Vincent MD, Leighl NB Biomarkers That Currently Affect Clinical Practice in Lung Cancer: EGFR, ALK, MET, ROS-1, and KRAS. Frontiers in oncology 2014; 4: 204 http://dx.doi.org/10.3389/fonc.2014.00204

[14] Soda M, Choi YL, Enomoto M, Takada S, Yamashita $Y$ Ishikawa $\mathrm{S}$, et al. Identification of the transforming EML4-ALK fusion gene in non-small-cell lung cancer. Nature 2007; 448(7153): 561-6. http://dx.doi.org/10.1038/nature05945

[15] Shaw AT, Kim DW, Nakagawa K, Seto T, Crino L, Ahn MJ, et al. Crizotinib versus chemotherapy in advanced ALK-positive lung cancer. N Engl J Med 2013; 368(25): 2385-94. http://dx.doi.org/10.1056/NEJMoa1214886

[16] Solomon BJ, Mok T, Kim DW, Wu YL, Nakagawa K, Mekhail $\mathrm{T}$, et al. First-line crizotinib versus chemotherapy in ALKpositive lung cancer. N Engl J Med 2014; 371(23): 2167-77. http://dx.doi.org/10.1056/NEJMoa1408440

[17] Purriños NDGNC. Biobancos y su importancia en el ámbito clínico y científico en relación con la investigación biomédica en Espanã. Reumatol Clin 2014; 10: 304-8. http://dx.doi.org/10.1016/i.reuma.2014.02.011

[18] Budimir D, Polasek O, Marusic A, Kolcic I, Zemunik T, Boraska V, et al. Ethical aspects of human biobanks: a 
systematic review. Croatian medical journal 2011; 52(3):

262-79.

http://dx.doi.org/10.3325/cmj.2011.52.262

[19] Hoeyer K. The ethics of research biobanking: a critical review of the literature. Biotechnology \& genetic engineering reviews 2008; 25: 429-52.

http://dx.doi.org/10.5661/bger-25-429

[20] Riegman $\mathrm{PH}$, de Jong BW, Llombart-Bosch A. The Organization of European Cancer Institute Pathobiology Working Group and its support of European biobanking infrastructures for translational cancer research. Cancer Epidemiol Biomarkers Prev 2010; 19(4): 923-6.

\section{http://dx.doi.org/10.1158/1055-9965.EPI-10-0062}

[21] OECD. Guidelines on human biobanks and genetic research databases 2009.

[22] Reis ST, Feitosa EB, Pontes-Junior J, Marin CC, Abe DK, Crippa A, et al. Tumor banks: the cornerstone of basic research in urology. International braz j urol : official journal of the Brazilian Society of Urology 2010; 36(3): 348-54. 